EPJ Web of Conferences 59, 02016 (2013)

DOI: $10.1051 /$ epjconf/20135902016

(C) Owned by the authors, published by EDP Sciences, 2013

\title{
Stability of target irradiation for high-repetition rate direct-drive facilities
}

\author{
Angelo Schiavi ${ }^{a}$, Stefano Atzeni and Alberto Marocchino \\ Dipartimento SBAI, Sapienza Università di Roma and CNISM, Via A. Scarpa, 14, \\ 00161 Roma, Italy
}

\begin{abstract}
The illumination of a spherical target for Inertial Confinement Fusion is studied in the direct-drive approach. Three main sources of laser parameter fluctuation are taken into account, and a statistical analysis of the illumination configuration has been carried out in order to assess the stability of the illumination, and the shot-to-shot fluctuation of the laser intensity pattern.
\end{abstract}

The illumination uniformity of the target is one of the key issues in direct-drive Inertial Confinement Fusion [1,2]. Any departure from perfect spherical symmetry in the compression drive could have detrimental effects on the implosion of the shell target, and could lead to ignition failure [3-5]. The problem becomes even more stringent when a high-repetition laser facility is envisaged, where target implosions take place several times per second in the reactor chamber. In this case the collocation of the fuel target at the centre of the compression drive might be difficult to control with accuracy and precision better than a few percent of target radius. We present a study of an illumination configuration [6], where the rms intensity non-uniformity is optimised taking into account three sources of departure from nominal parameters, i.e. the laser pointing error, the beam-to-beam power imbalance, and the target positioning. It is found that the fluctuation of the illumination asymmetry is a key parameter for assessing the stability of operation in high-repetition rate mode. Moreover the target positioning error arising from the injection system could be the dominant source of illumination asymmetry in a fusion reactor.

The analysis procedure outlined in the paper is of general validity, but for the sake of clarity, the work presented is based on the study of a particular irradiation pattern, consisting of 48 different beamlines distributed on a sphere. This configuration is the one envisaged for the laser system proposed within the HiPER framework, which is aimed at the demonstration of a burst-mode fusion reactor exploiting advanced ignition schemes, such as the Shock Ignition approach or the Fast Ignitor scheme [7]. The beams are grouped in 6 cones symmetrically distributed around the equator in the polar angle $\theta$. Within each cone, the beams are evenly distributed around the symmetry axis with equal spacing in the azimuthal angle $\phi$ [8]. The details of the configuration are summarised in the left panel of Figure 1. In addition to the beam centroids on the irradiation sphere, the main parameters that define the laser configuration are the time and space profile of the laser power carried by each beam. We describe the intensity spatial profile as $I(r)=I_{0} \exp \left[-(r / \Delta)^{m}\right]$, where $r$ is the radial distance for the beam axis, $I_{0}$ is the peak intensity, $\Delta$ is the effective waist of the beam, and $m$ is the super-Gaussian exponent. With $R_{0}$ we indicate the initial outer radius of the target.

The average intensity on the target is given by $<\mathrm{I}>=\int I(\theta, \phi) d \Omega / \int d \Omega$, where the integration is carried out over the whole solid angle, and $I(\theta, \phi)$ is the local intensity on the target surface.

\footnotetext{
${ }^{a}$ e-mail: angelo.schiavi@uniroma1.it
}

This is an Open Access article distributed under the terms of the Creative Commons Attribution License 2.0, which permits unrestricted use, distribution, and reproduction in any medium, provided the original work is properly cited. 

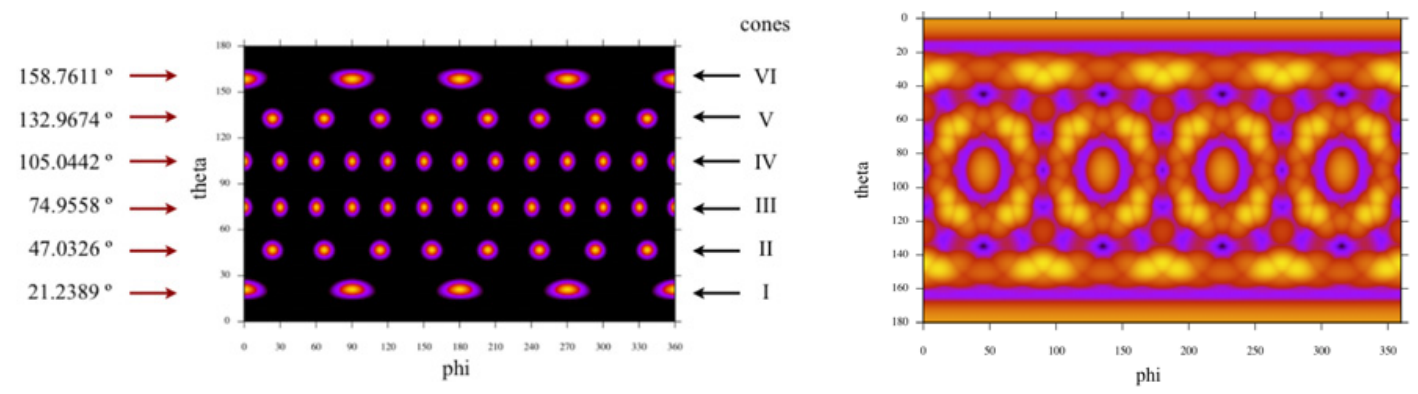

Figure 1. Direct-drive 48-beam irradiation pattern (left) and intrinsic intensity pattern (right).
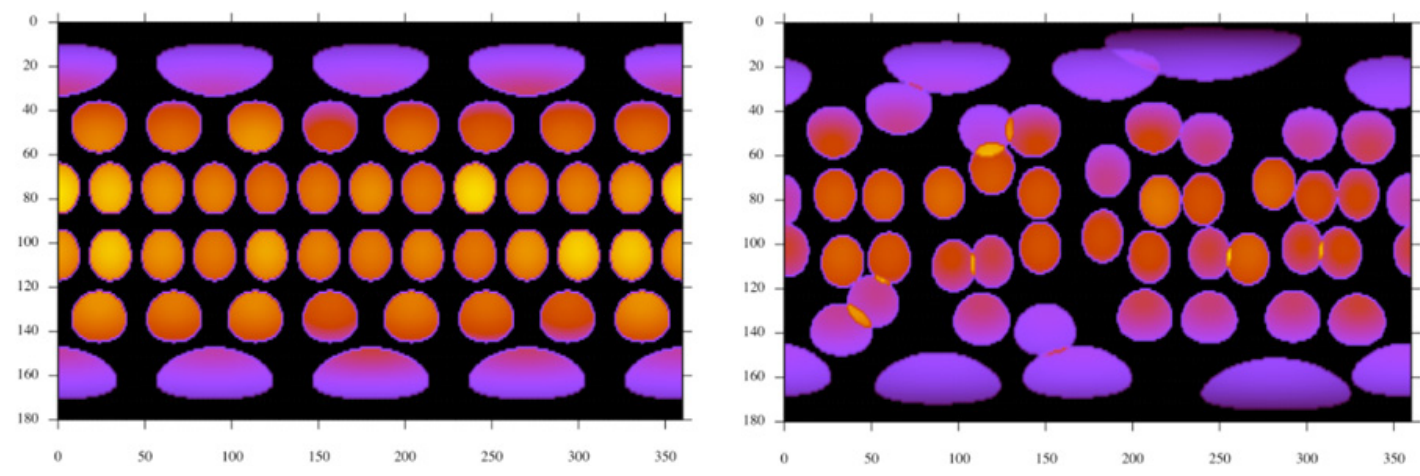

Figure 2. Beam-to-beam power imbalance (left) and pointing error (right) (exaggerated for demonstration).

A measure of the illumination uniformity is obtained by calculating the root mean square deviation defined as $\sigma_{r m s}^{2}=\int(I-<I>)^{2} d \Omega / \int d \Omega$. We use the relative deviation $\sigma=\sigma_{r m s} /<\mathrm{I}>$ as a measure of the uniformity of illumination.

The initial proposal for the HiPER beam configuration $[9,10]$ is described by $m=2.04$ and $\Delta=0.61 R_{0}$, and represented in the $(m, \Delta)$ plots shown in this work as a red circle (see for instance Figure 4). The intensity pattern corresponding to such a choice of parameters is shown in Figure 1, and is characterised by a non-uniformity $\sigma_{0}=0.15 \%$. This point in the irradiation space corresponds to a local minimum in illumination asymmetry $\sigma$, but is characterised by a high sensitivity to laser system errors (see [6] and references therein).

We considered in our analysis three main sources of departure of laser parameters from nominal: the beam-to-beam power imbalance (PI), the laser pointing error (PE), and the target positioning (TP) at the interaction chamber centre. The PE and the TP values are expressed as percentage of the capsule outer radius. A graphical representation of the PI and PE is given in Figure 2: here the width of the beams is smaller than the target radius (i.e. $\Delta \simeq 0.1 R_{0}$ ), and the level of fluctuation is exaggerated for presentation sake. The actual beam profile was instead used in Figure 3, where a 5\% TP error is introduced. Here a dipole moment is clearly visible, superimposed to the intrinsic intensity pattern of Figure 1. For capsules designed for ICF having typically a radius of about a $\mathrm{mm}$, this initial displacement corresponds to a situation where the target is shifted $50 \mu \mathrm{m}$ away from the laser system centre. Collocation of the target at high-repetition rate (a few Hz) in a foreseen ICF reactor with a precision better than $50 \mu \mathrm{m}$ is presently considered very critical. We developed a numerical code for computing the illumination asymmetry of a given beam configuration, and we used it for assessing the average $\sigma$ and its rms deviation $\delta \sigma$ for a large number of irradiation patterns, varying the power level, the beam offset and the position of the target within a given level of PI, PE and TP. The main result is presented in Figure 4, where the isolines of 


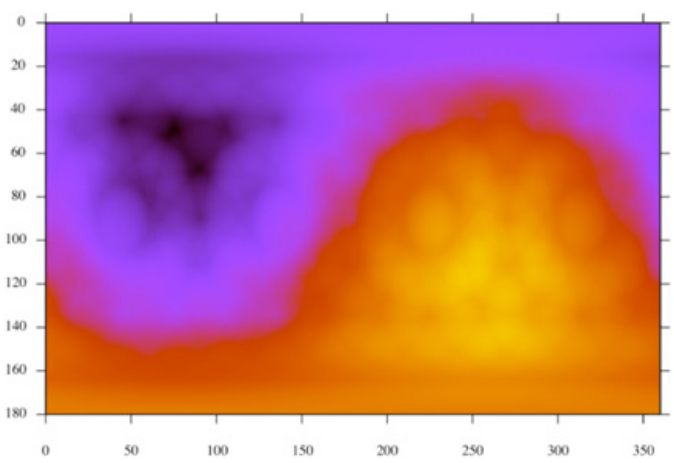

Figure 3. Target positioning error at $5 \%$ level.
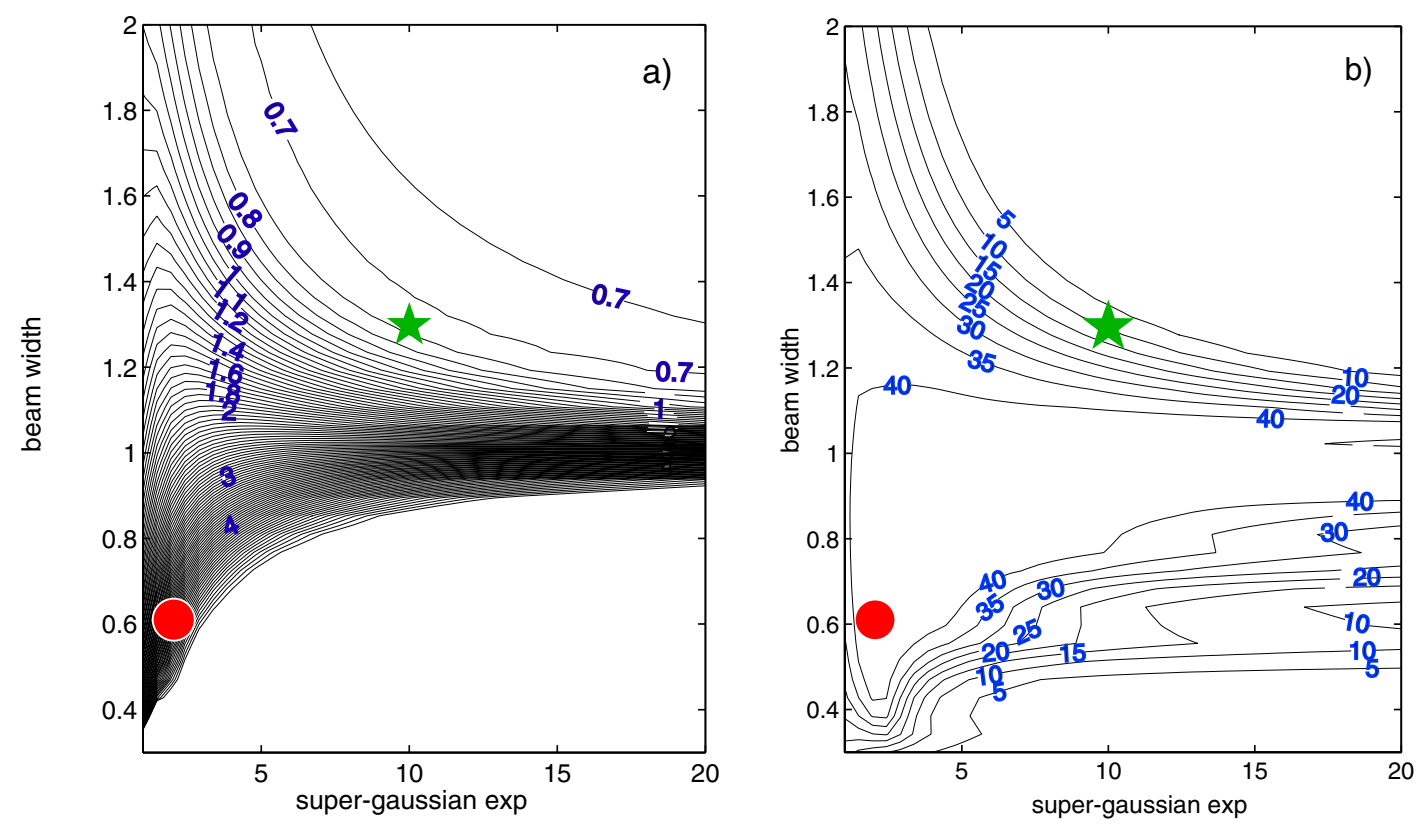

Figure 4. Isolines of average illumination non-uniformity $\sigma$ (left) and relative fluctuation $\delta \sigma$ (right).

intensity non-uniformity are plotted on the left for a typical case where $\mathrm{PI}=1 \%, \mathrm{PE}=5 \%$ and $\mathrm{TP}=5 \%$. The red circle marks the original working point for HiPER, which does not lay on a local minimum island, but on a steep slope, as a consequence of the shot-to-shot errors. The right panel of the same figure presents the relative fluctuation of the $\sigma$, measuring the level of shot-to-shot variation in the quality of the illumination pattern. It can be seen that the red circle corresponds to fluctuation of more than $40 \%$ in $\sigma$, indicating a very unstable irradiation configuration. For increasing the reliability of the laser compression drive, it is clearly convenient to shift the design point of the laser system towards regions of higher stability. An increase in beam diameter well above the target radius, and a flattening of the beam wings by increasing the super-gaussian exponent $m$ will reduce the sensitivity to TP, which appears to be the critical source of shot-to-shot intensity fluctuations. A tentative point (green star) was obtained in [6], emerging as a good compromise between shot-to-shot stability and moderate increase in laser energy requirements as a consequence of beam waist growth with respect to the original design point. 


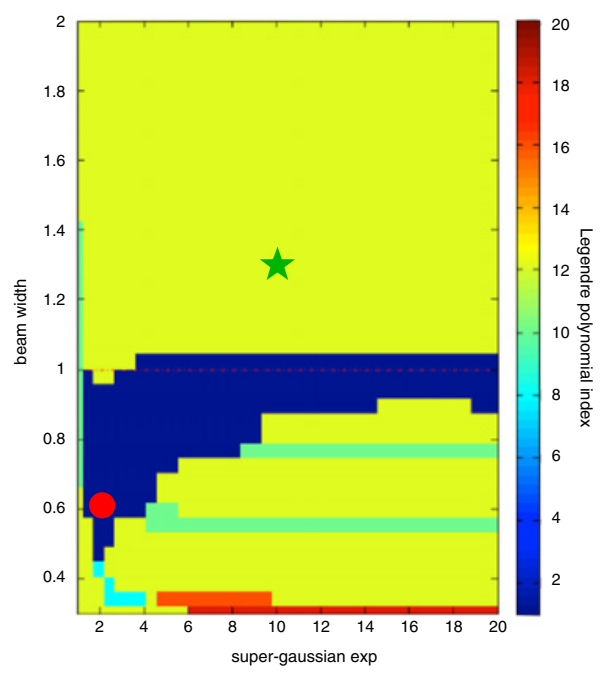

Figure 5. Dominant Legendre mode for each illumination configuration.

An analysis in Legendre polynomials was carried out for the same set of laser facility errors and is presented in Figure 5. There the dark blue colour corresponds to a dominant mode $l=1$ in the intensity pattern, blueprint of target displacement as seen before. The red circle lays in a region dominated by the TP error. The green star point instead is inside the region dominated by the $l=12$ mode, which is originated by the 48-beam irradiation pattern. This result has important implications for subsequent studies of target implosion: it states that the stability analysis carried out so far $[9,11]$ in $2 \mathrm{D}$ cylindrical symmetry and based on Legendre polynomials expansion of the laser drive could be reinterpreted and applied to the new working point. Moreover, in these illumination conditions, the level of TP could be considered as a perturbation to the intrinsic illumination pattern, and therefore should not dramatically affect the implosion of the capsule. Finally, the intensity pattern for the green star point is more stable, and is related to the actual design of the laser facility. At the same time the effective illumination $\sigma$ is less prone to fluctuations due to laser operation uncertainties. This is a very important aspect indicating that a "stable" working point for a laser facility can be chosen in such a way that target modeling and implosion simulations can be carried out before the actual experimental campaign with a good degree of confidence. Clearly the outlined procedure can be applied to any multi-beam illumination pattern, e.g. the M48 configuration proposed by [12] which has the important advantage over the one presented here that the beams are not aligned head-on.

The presented results have been obtained using simple illumination calculations, which allow for a large parametric scan in beam configuration space. Thousands of laser-shots were simulated in order to acquire significant statistics, essential for assessing the illumination $\sigma$ fluctuations. Presently we are extending our analysis using 2D hydrodynamic simulations of the capsule irradiation coupled to a fully $3 \mathrm{D}$ raytracing package for laser energy deposition in the plasma.

This work was partially supported by the Italian MIUR project PRIN 20072KW45J and by the HiPER project and Preparatory Phase Funding Agencies (EC, MSMT and STFC).

\section{References}

[1] J. D. Lindl, Inertial Confinement Fusion: the Quest for Ignition and Energy Gain Using Indirect Drive (Springer, New York, 1998)

[2] S. Atzeni, J. Meyer-ter-Vehn, The Physics of Inertial Fusion (Clarendon, Oxford, 2004) 


\section{IFSA 2011}

[3] R. L. McCrory, C. P. Vernon, Inertial Confinement Fusion, Proceedings of the Course and Workshop, Varenna, Italy 6-16 Sept. 1988 (Compositori-SIF, Bologna, 1989)

[4] S. Atzeni, Europhys. Lett. 7, 639 (1990)

[5] R. Kishony, D. Shvarts, Phys. Plasmas 8, 4925 (2001)

[6] A. Schiavi, S. Atzeni, A. Marocchino, EPL 94, (2011) 35002

[7] M. Dunne, Nature Phys. 2, 2 (2006)

[8] J. Xiao, B. Lu, J. Opt. 29, 282 (1998)

[9] L. Hallo et al., Plasma Phys. Control. Fusion 51, 014001 (2009)

[10] S. Atzeni S. et al., Nucl. Fusion 49, 055008 (2009)

[11] S. Atzeni, A. Schiavi, A. Marocchino, Plasma Phys. Control. Fusion 53, 035010 (2011)

[12] M. Murakami, N. Sarukura, H. Azechi, M. Temporal, A. J. Schmitt, Phys. Plasmas 17, 082702 (2010) 